Sharif University of Technology
Scientia Iranica
SCIENTIA

\title{
MHD mixed convection boundary layer flow of a Casson fluid bounded by permeable shrinking sheet with exponential variation
}

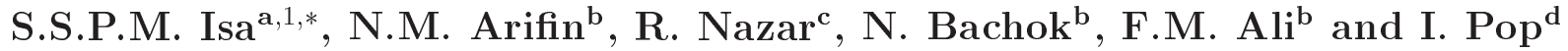 \\ a. Institute for Mathematical Research, Universiti Putra Malaysia, 43400 UPM Serdang, Selangor, Malaysia. \\ b. Department of Mathematics, Universiti Putra Malaysia, 43400 UPM Serdang, Selangor, Malaysia. \\ c. School of Mathematical Sciences, Faculty of Science \& Technology, Universiti Kebangsaan Malaysia, 43600 UKM Bangi, \\ Selangor, Malaysia. \\ d. Department of Mathematics, Babeş-Bolyai University, R-400084 Cluj-Napoca, Romania.
}

Received 20 April 2015; received in revised form 14 December 2015; accepted 9 May 2016

\section{KEYWORDS}

Casson fluid;

MHD;

Mixed convection;

Exponentially

shrinking sheet.

\begin{abstract}
A review was carried out on the exponentially permeable shrinking sheet and how it influenced the magnetohydrodynamic (MHD) mixed convection boundary layer flow of a Casson fluid. The boundary layer equations in the form of partial differential equations were transformed into the ordinary differential equations by using the similarity transformation. Subsequently, shooting technique is used to provide solutions for the ordinary differential equations. Different factors related to the flow and heat are indicated by the attained results as well as graphs. Moreover, 4 solutions are presented graphically. Also, the numerical calculations exhibit that the Casson fluid parameter, $\varepsilon$, buoyancy parameter, $\lambda$, and suction parameter, $s$, would significantly affect the characteristics of flow and thermal boundary layers of a Casson fluid.

(C) 2017 Sharif University of Technology. All rights reserved.
\end{abstract}

\section{Introduction}

There is great benefit to the industry by exploring the convection features of non-Newtonian fluids, such as liquid-liquid extractors [1], catalytic reactors [2], blood plasmaphosresis devices [3], and the filtration devices [4]. The Casson fluid is commoly known in the midst of non-Newtonian fluids, which was introduced by Casson [5]. This fluid is a good approximation of many substances, such as blood, molten chocolate, foams, and cosmetics, among others. As a consequence, numerous researchers have investigated the features

1. Present address: Centre of Foundation Studies for Agricultural Science, Universiti Putra Malaysia, 43400 UPM Serdang, Selangor, Malaysia.

*. Corresponding author. Tel.: +603-8946 6998; Fax: +603-8946 6997

E-mail address: suzi_isa@yahoo.com (S.S.P.M.Isa) of Casson fluid flow ([6-9] etc.). Besides, Boyd et al. [10] studied two types of flow, namely, steady and oscillatory flows, in straight and curved pipe geometries. In their research, Boyd et al. [10] used the Carreau-Yasuda and Casson fluids.

There are various benefits to the engineering process by studying boundary layer flow and transmission of heat instigated by a shrinking sheet, for example glass casting, wire drawing, glass fibre manufacturing, and removal of polymer sheet. Goldstein [11] mentioned that the flow of fluid caused by coiling sheet was fundamentally a rearward flow, which was the reverse of the flow caused by an extending sheet. A study by Miklavčič and Wang [12] found that analytical solution to two-dimensional viscous flows was due to the shrinking sheet with suction; the researchers reported that the flow over the shrinking sheet was sustained by the suction. Mahapatra and Nandy [13] 
agreed with study results of Miklavčič and Wang [12] who came to the conclusion that the steady flow as a result of the shrinking sheet was unable to avoid the impact of suction. An evaluative study examined the two-dimensional flow together with heat transfer of viscous fluid towards a shrinking sheet was performed by Wang [14]. He proved that the solutions obtained were non-unique for insignificant value of the shrinking factor.

Magyari and Keller [15] were the pioneers who studied the flow and thermal boundary layers confined by an exponential stretching sheet. However, in the case related to a shrinking sheet with exponential velocity, Bhattacharyya [16] was the first person to explore the related field. His study results demonstrated the presence of twofold solutions in the suction. Some of the latest dual nature studies have involved exponentially stretching/shrinking sheet $[17,18]$. However, recent studies have been reported on the boundary layer flow and the transfer of heat in non-Newtonian fluid towards a stretching/shrinking sheet, which involves the concept of magnetohydrodynamic (MHD) stagnation-point flow $[19,20]$ and unsteady state [21$23]$.

Recently, the presence of multiple solutions has been confirmed by certain researchers [24-29]. A recent study by Rosca and Pop [29] came up with engaging facts to provide evidence for the assumption of dual numerical solutions. The researchers [29] carried out a stability examination and reported that the solutions found on the upper branch (primary solution) were linearly stable, whereas those on the lower branch (secondary solution) were linearly unstable. The physically relevant solution was related to the steady solution. The discovery of triple solutions is quite new and of interest. Therefore, in the event that Newtonian fluid is able to pass through the shrinking sheet with an increasingly exponential velocity, there exists formation of the triple solution because of the impact of the flow's buoyancy force along with traits associated with the flow and heat transfer [30]. Subsequently, Rohni et al. [31] proved that triple solutions also occur when the stagnation point is implemented in the system of Newtonian fluid. Recently, an extension of the work by Rohni et al. [30] was carried out by Isa et al. [32]. Accordingly, the researchers [32] were able to get the quad solutions because of the addition of a magnetic field in the fluid flow's system developed by Rohni et al. [30].

The theoretical study in the Casson fluid model is reportedly based on the features of the flow and heat transmission. A unique solution is illustrated for linear stretching sheet case, whereas dual solutions exist for the problem of magnetohydrodynamic linear shrinking sheet [33-36]. MHD Casson fluid flows in a system of three-dimensional coordinates related to the stretching sheet in linear velocity, as reported by Nadeem et al. $[37,38]$. A numerical evaluation was carried out on the challenges associated with using the Casson fluid flow in a non-Darcy porous medium commencing at a horizontal circular cylinder surrounded by partial slips condition [39-40]. The Casson fluid flow over a sheet, which is stretched with exponential variation, is taken into account by Pramanik [41], Raju et al. [42], and Animasaun et al. [43]. The permeable shrinking sheet, which has an exponential velocity and contributes a significant influence on the characteristics of MHD Casson fluid flow, has been analyzed by Nadeem et al. [44]. However, they only analyzed the features of Casson flow, and heat transfer was not taken into account. Later, Haq et al. [45] used the same model as that of Nadeem et al. [44], but with the additional effect of a convective boundary condition.

Inspired by the previous research, this study expands the work by Isa et al. [32] in an effort to offer replacement of the viscous fluid with the nonNewtonian Casson fluid. This study is also an extension of the study by Nadeem et al. [44] by including an analysis of heat transfer characteristics in Casson flow over a sheet, which has an exponential shrinking rate. The results obtained by the present study reveal that the temperature and flow fields are controlled by Casson fluid parameter, $\varepsilon$, mixed convection/buoyancy parameter, $\lambda$, and suction parameter, $s$.

\section{Mathematical formulation}

Figure 1 illustrates the steady, laminar, two-dimensional boundary layer flow of an incompressible Casson fluid over an exponentially shrinking sheet. The uniform temperature of the ambient fluid is $T_{\infty}$ and the surface temperature is $T_{w}$. Assisting flow case is

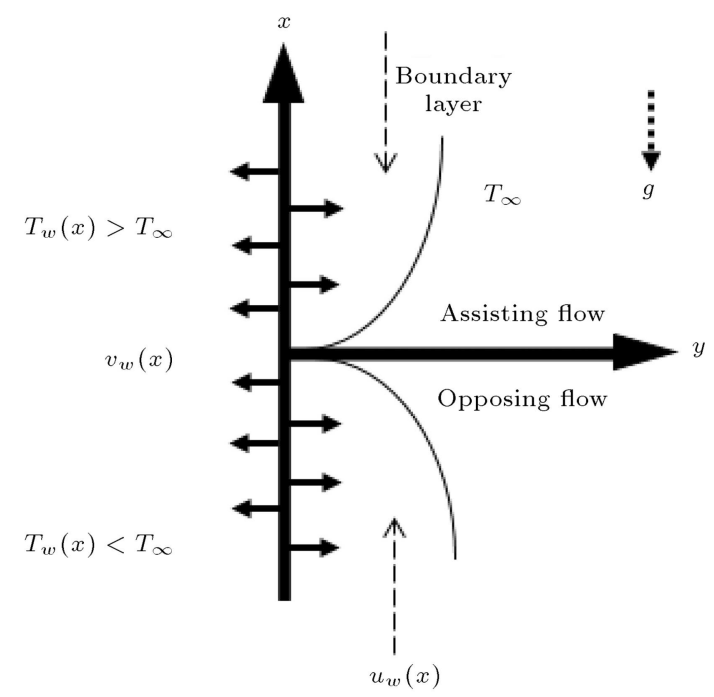

Figure 1. Physical model and coordinate system of a Casson fluid. 
denoted by $T_{w}>T_{\infty}$, whereas $T_{w}<T_{\infty}$ corresponds to the opposing flow case. There is application of a transverse magnetic field, $B(x)$, in a tangential line perpendicular to the plane $y=0$. Nakamura and Sawada [46] studied the status of an isotropic Casson fluid along with an incompressible one in a rheological equation. This equation is shown below:

$$
\tau_{i j}= \begin{cases}2\left(\mu_{B}+\frac{p_{y}}{\sqrt{2 \pi}}\right) e_{i j}, & \pi>\pi_{c} \\ 2\left(\mu_{B}+\frac{p_{y}}{\sqrt{2 \pi_{c}}}\right) e_{i j}, & \pi<\pi_{c}\end{cases}
$$

where $\mu_{B}$ is plastic dynamic viscosity of the nonNewtonian fluid, $p_{y}$ is the yield stress of fluid, $\pi=$ $e_{i j} e_{i j}$ and $e_{i j}$ are the $(i, j)^{i h}$ components of the deformation rate, $\pi$ is the square of the component of the deformation rate, and $\pi_{c}$ is a critical value of $\pi$ based on the non-Newtonian model. From Eq. (1), plastic dynamic viscosity is $\mu_{B}=1 / 2\left(\tau_{i j} / e_{i j}\right)-p_{y} / \sqrt{2 \pi}$, kinematic viscosity is $\nu=\mu_{B} / \rho$ where $\rho$ is the fluid density, and Casson parameter is formulated as $\gamma=$ $\mu_{B}\left(\sqrt{2 \pi_{c}} / p_{y}\right)$.

The governing boundary layer equations can be written as:

$$
\begin{aligned}
& \frac{\partial u}{\partial x}+\frac{\partial v}{\partial y}=0 \\
& u \frac{\partial u}{\partial x}+v \frac{\partial u}{\partial y}=\nu\left(1+\frac{1}{\gamma}\right) \frac{\partial^{2} u}{\partial y^{2}}+g \beta\left(T-T_{\infty}\right) \\
& -\frac{\sigma B^{2}(x)}{\rho} u \\
& u \frac{\partial T}{\partial x}+v \frac{\partial T}{\partial y}=\alpha \frac{\partial^{2} T}{\partial y^{2}},
\end{aligned}
$$

where $u$ and $v$ are the velocity elements in the $x$ and $y$ directions, respectively, $g$ is the acceleration due to gravity, $\beta$ is thermal expansion coefficient, $\sigma$ is electrical conductivity (assumed constant), $\alpha$ is thermal diffusivity, and $T$ is the fluid temperature. In Eq. (3), we choose the magnetic field $B(x)=B_{0} e^{(x / 2 L)}$, where $B_{0}$ is the constant magnetic field. Therefore, the related boundary conditions are:

$$
\begin{array}{ll}
u=u_{w}(x)=-U_{w} e^{(x / L)}, & v=v_{w}(x), \\
T_{w}(x)=T_{\infty}+T_{0} e^{(2 x / L)} & \text { at } \quad y=0, \\
u \rightarrow 0 \quad T \rightarrow T_{\infty} & \text { as } \quad y \rightarrow \infty,
\end{array}
$$

where $U_{w}>0$ is the shrinking velocity and $v_{w}(x)<0$ is for suction.
Non-dimensional variables used in this problem are:

$$
\begin{aligned}
& \eta=y\left(\frac{U_{w}}{2 \nu L}\right)^{1 / 2} e^{(x / 2 L)} \\
& \Psi=\left(2 \nu L U_{w}\right)^{1 / 2} f(\eta) e^{(x / 2 L)} \\
& \theta(\eta)=\frac{T-T_{\infty}}{T_{w}-T_{\infty}}
\end{aligned}
$$

where stream function, $\Psi$, is defined as $u=\partial \Psi / \partial y$ and $v=-\partial \Psi / \partial x$.

Substituting Eq. (6) into Eqs. (2)-(4), the governing equations are obtained using the dimensionless functions $f(\eta)$ and $\theta(\eta)$ as follows:

$$
\begin{aligned}
& \left(1+\frac{1}{\gamma}\right) f^{\prime \prime \prime}+f f^{\prime \prime}-2\left(f^{\prime}\right)^{2}-M^{2} f^{\prime}+2 \lambda \theta=0 \\
& \frac{1}{\operatorname{Pr}} \theta^{\prime \prime}+f \theta^{\prime}-4 f^{\prime} \theta=0
\end{aligned}
$$

The boundary conditions are reduced to:

$$
\begin{aligned}
& f^{\prime}(\eta)=-1, \quad f(\eta)=s, \quad \theta(\eta)=1 \quad \text { at } \quad \eta=0, \\
& f^{\prime}(\eta) \rightarrow 0, \quad \theta(\eta) \rightarrow 0, \quad \text { as } \quad \eta \rightarrow \infty,
\end{aligned}
$$

where primes denote differentiation with respect to $\eta, M^{2}=2 \sigma B_{0}^{2} L / \rho U_{w}$ is the Hartman number (also defined as magnetic field parameter), $\operatorname{Pr}=\nu / \alpha$ is the Prandtl number, $s=-\left(2 L / v U_{w}\right)^{1 / 2}\left(1 / e^{x / 2 L}\right) v_{w}(x)>$ 0 is the suction parameter, and $\lambda=\mathrm{Gr} / \mathrm{Re}^{2}$ is the constant mixed convection parameter, where $\mathrm{Gr}=$ $g \beta_{T} T_{0} L^{3} / \nu^{2}$ is the Grashof number and $\operatorname{Re}=U_{w} L / \nu$ is the Reynolds number. It is mainly noticeable that $\lambda>0, \lambda<0$, and $\lambda=0$ refer to the assisting flow, opposing flow, and forced convection flow, respectively.

This paper will focus on the following physical quantities: the skin friction coefficient $C_{f}$ as well as local Nusselt number $\mathrm{Nu}_{x}$ :

$$
C_{f}=\frac{\tau_{w}}{\rho u_{w}^{2}(x)}, \quad \mathrm{Nu}_{x}=\frac{L}{T_{w}-T_{\infty}}\left(-\frac{\partial T}{\partial y}\right)_{y=0} .
$$

Substituting Eq. (6) into Eq. (10), we get:

$$
\begin{aligned}
& C_{f}\left(2 \operatorname{Re}_{x}\right)^{1 / 2} e^{(-3 x / 2 L)}=\left(1+\frac{1}{\gamma}\right) f^{\prime \prime}(0), \\
& \mathrm{Nu}_{x}\left(\frac{2}{\operatorname{Re}_{x}}\right)^{1 / 2} e^{(-x / 2 L)}=-\theta^{\prime}(0),
\end{aligned}
$$

where $\operatorname{Re}_{x}=x u_{w}(x) / \nu$ is a local Reynolds number. It is meaningful to emphasize that the problem of nonNewtonian Casson fluid can be reduced to the case of Newtonian fluid when $\gamma \rightarrow \infty$. 


\subsection{Numerical method for solution}

The shooting technique gives the solutions for the boundary value problems in Eqs. (7), (8), and (9) by changing them into an initial value problem. The fourth-order Runge-Kutta integration scheme is applied to solve the initial value problem. We set:

$$
\begin{aligned}
& f^{\prime}=f p, \quad f p^{\prime}=f p p \\
& f p p^{\prime}=\frac{-f(f p p)+2(f p)^{2}+M^{2} f p-2 \lambda \theta}{(1+1 / \gamma)} \\
& \theta^{\prime}=\theta p, \quad \theta p^{\prime}=-\operatorname{Pr}(f \theta p)+4 \operatorname{Pr}(f p \theta),
\end{aligned}
$$

with the boundary conditions:

$$
\begin{array}{lll}
f p(0)=-1, & f(0)=s, & \theta(0)=1, \\
f^{\prime}(\eta) \rightarrow 0 & \theta(\eta) \rightarrow 0 & \text { as } \quad \eta \rightarrow \infty .
\end{array}
$$

Firstly, the values of the physical parameters included in Eqs. (12)-(14) are fixed. In order to carry out the integration in Eqs. (12) and (13) as an initial value problem, we assume initial values for $f p p(0)$, i.e. $f^{\prime \prime}(0)$ and $\theta p(0)$, i.e. $\theta^{\prime}(0)$. We also assume a suitable finite value for $\eta \rightarrow \infty$ say, $\eta_{\infty}$. Then, we calculate the values of $f^{\prime}\left(\eta_{\infty}\right)$ and $\theta\left(\eta_{\infty}\right)$ by adjusting the assumed values of $f^{\prime \prime}(0)$ and $\theta^{\prime}(0)$ to give a better approximation of the solution. Basically, the assumed values of $\eta_{\infty}, f^{\prime \prime}(0)$ and $\theta^{\prime}(0)$ satisfy the boundary conditions in Eq. (14) $\left(f^{\prime}\left(\eta_{\infty}\right)=0\right.$ and $\left.\theta\left(\eta_{\infty}\right)=0\right)$. This steps is repeated with higher values of $\eta_{\infty}$ until 2 consecutive values of $f^{\prime \prime}(0)$ and $\theta^{\prime}(0)$ vary significantly by a specified value. Finally, the most appropriate value of $\eta_{\infty}$ is selected, which is the largest $\eta_{\infty}$ that satisfies the solution.

\section{Results and discussion}

A shooting method is used to solve the nonlinear ordinary differential equations (7) and (8), subject to boundary condition (9). Since the problem formulation can be transformed to the case of Newtonian fluid by setting $\gamma \rightarrow \infty$, the accuracy of the applied numerical method is tested by a direct comparison of the values of the skin friction coefficient when $\gamma=100000$, with those reported by Isa et al. [32] for $\gamma=0$. The results are presented in Table 1 . It is evident that the values obtained for the skin friction coefficient agree with values attained by Isa et al. [32]. In conclusion, our numerical method presented here could be used with great confidence for further investigation. To illustrate the flow and heat transfer characteristics of the opposing flow case, the variations of the skin friction coefficient, $C_{f}\left(2 \operatorname{Re}_{x}\right)^{1 / 2} e^{(-3 x / 2 L)}$, local Nusselt number, $\mathrm{Nu}_{x}\left(2 / \operatorname{Re}_{x}\right)^{1 / 2} e^{(-x / 2 L)}$, velocity profile, $f^{\prime}(\eta)$, and temperature profile, $\theta(\eta)$, are depicted. Figures 2 and 3 show the variations of skin friction coefficient

\begin{tabular}{|c|c|c|c|}
\hline \multicolumn{4}{|c|}{$C_{f}\left(2 \mathrm{Re}_{x}\right)^{1 / 2} e^{(-3 x / 2 L)}$} \\
\hline \multirow{3}{*}{$\lambda$} & \multirow{3}{*}{$\begin{array}{c}\text { Solution } \\
\text { profiles }\end{array}$} & \multicolumn{2}{|l|}{$\gamma$} \\
\hline & & 0 & $\infty$ \\
\hline & & Isa et al. [26] & present \\
\hline \multirow{4}{*}{-0.5} & First & 3.3616 & 3.3615 \\
\hline & Second & -3.2002 & -3.2001 \\
\hline & Third & -6.7088 & -6.7088 \\
\hline & Fourth & -0.4698 & -0.4699 \\
\hline \multirow{4}{*}{1} & First & 4.2806 & 4.2805 \\
\hline & Second & -2.7500 & -2.7499 \\
\hline & Third & -3.1944 & -3.1943 \\
\hline & Fourth & 0.6641 & 0.6641 \\
\hline
\end{tabular}

Table 1. Comparison of the values of skin friction coefficient $C_{f}\left(2 \operatorname{Re}_{x}\right)^{1 / 2} e^{(-3 x / 2 L)}$ with those of Isa et al. [26] for Newtonian fluid and present results for a very large Casson parameter.



Figure 2. Variations of skin friction coefficient $C_{f}\left(2 \operatorname{Re}_{x}\right)^{1 / 2} e^{(-3 x / 2 L)}$ with the suction parameter $s$ for different values of $M^{2}$ when $\operatorname{Pr}=1, \gamma=2$, and $\lambda=-1$.

and local Nusselt number, respectively, with changes in the rate of suction $s$ for different values of $M^{2}$. It has been evidenced that $M^{2}$ increases the skin friction coefficient as well as the local Nusselt number according to the first solution. On the other hand, other profiles show opposite trends. The first and second solutions are connected at the point $s=s_{c}$, and these two profiles exist at $s>s_{c}$. The third and fourth solutions occur for all values of the suction parameter $s$. The effects of the magnetic field on the skin friction coefficient and the local Nusselt number were found to be more pronounced for a larger $s$. For the third and fourth solution profiles, the values of skin friction coefficient and local Nusselt number were close to each other when the rate of suction was reduced. As it is clear, when we refer to Figure 2 for the first solution, a large value of $s$ produced a highly positive value of the skin friction 
coefficient. On the other hand, it was found that the third solution gave large negative values of the skin friction coefficient when $s$ was very large. It is evident from Figure 3 that a high rate of suction led to an increment of the local Nusselt number for the first, third, and fourth solution profiles. We can conclude that the rate of heat transfer in the third and fourth solutions decreased with a decrease in $s$ until the rate of suction was close to zero. These two profiles (third and fourth solutions) showed a very slight difference in the values of the local Nusselt number when the wall tended to become impermeable.

The dimensionless velocity profile when $\lambda=-1$ is depicted in Figure 4, which shows that the third

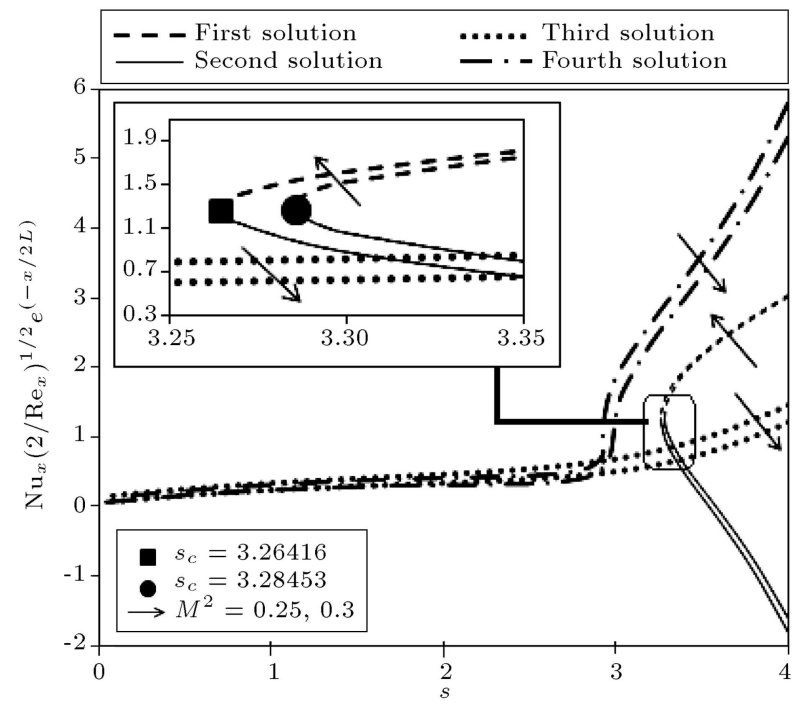

Figure 3. Variations of local Nusselt number $\mathrm{Nu}_{x}\left(2 / \operatorname{Re}_{x}\right)^{1 / 2} e^{(-x / 2 L)}$ with the suction parameter $s$ for different values of $M^{2}$ when $\operatorname{Pr}=1, \gamma=2$, and $\lambda=-1$.

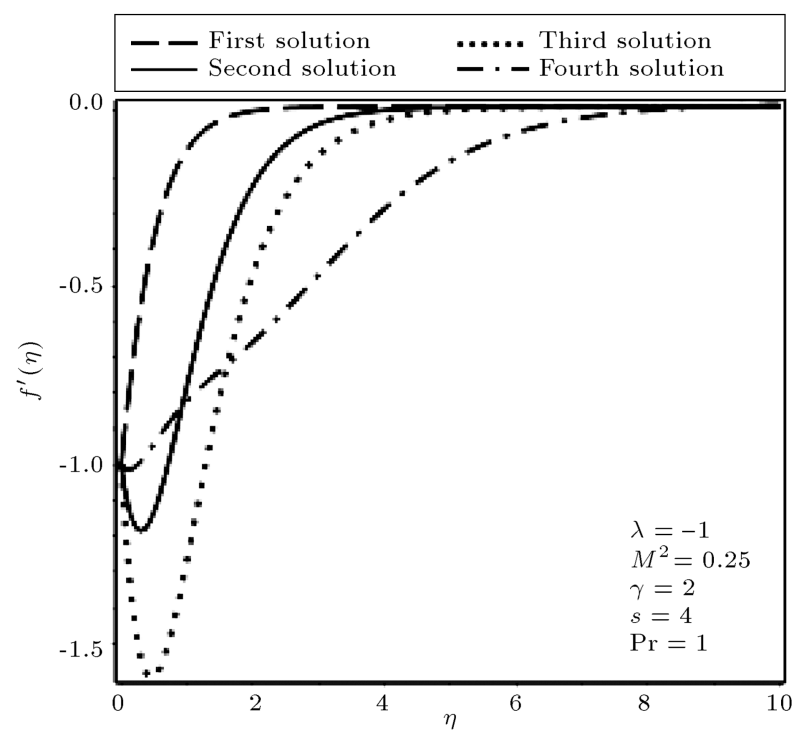

Figure 4. Velocity profiles when $\lambda=-1$.

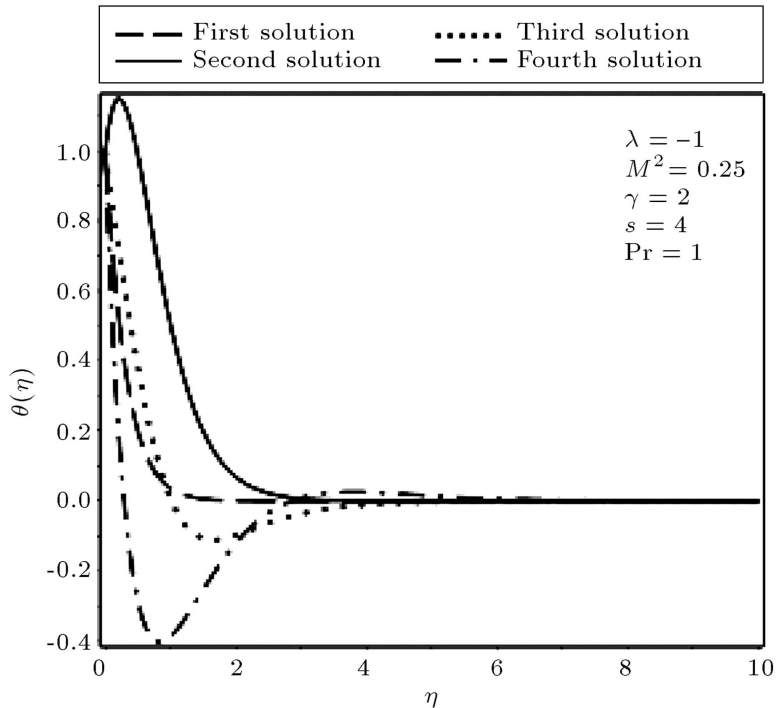

Figure 5. Temperature profiles when $\lambda=-1$.

solution had the largest magnitude of velocity when the point of the fluid was close to the shrieked wall. After this, negative $f^{\prime}(\eta)$ in all profiles tended to attain a constant zero velocity. It implies that there was reduction in the magnitude of velocity that was in the reverse direction, until the flow ended. Figure 5 shows the temperature distribution $\theta(\eta)$ for $\lambda=-1$. In the third and fourth solution profiles, the temperature $\theta(\eta)$ at the point initially decreased and then became negative. Subsequently, the temperature started to increase when the distance from the shrieked wall and the body of the fluid became larger. The second solution showed the presence of a peak for a small value of $\eta$, indicating that the maximum temperature occurs in the fluid at a point near the shrieked wall. The enhancement of the temperature difference between the shrieked wall and the adjacent fluid was indicated by the increment of temperature peak value. As a result, the heat transfer process from the shrieked wall to the ambient fluid was enhanced.

The skin friction coefficient and the local Nusselt number for several values of $M^{2}$, for $\operatorname{Pr}=1, \gamma=2$, and $\lambda=1$, are shown in Figures 6 and 7 , respectively. From Figure 6 , it is clear that the impact of the magnetic field was to enhance the rate of skin friction coefficient in the first and third solutions. On the other hand, the opposite trend occurred in the other 2 profiles. Moreover, the local Nusselt number increased in the first, second, and fourth solutions with the effect of the magnetic field. When $M^{2}=0.25$, the critical suction parameter $s_{c}$ connected the third and second profiles, but the third solution profile was connected with the fourth solution at the point $s_{c}$ when $M^{2}=0.3$. The occurrence of the first solution profile was observed for various values of $M^{2}$ and $s$. With regard to the variations of skin friction coefficient and local Nusselt number, the second solution profile continued until 




Figure 6. Variations of skin friction coefficient $C_{f}\left(2 \operatorname{Re}_{x}\right)^{1 / 2} e^{(-3 x / 2 L)}$ with the suction parameter $s$ for different values of $M^{2}$ when $\operatorname{Pr}=1, \gamma=2$, and $\lambda=1$.

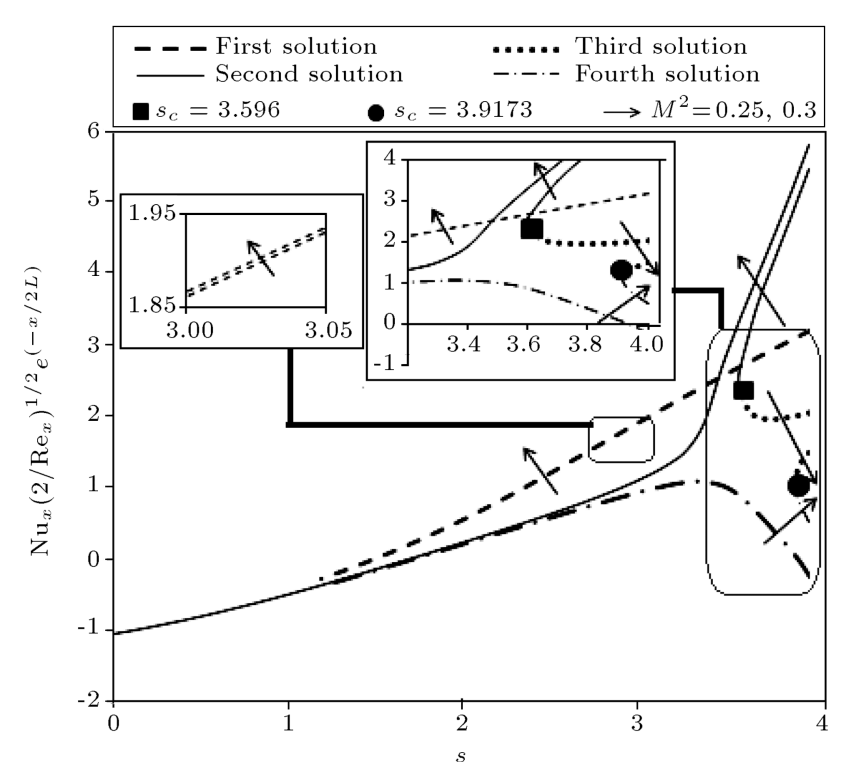

Figure 7. Variations of local Nusselt number $\mathrm{Nu}_{x}\left(2 / \operatorname{Re}_{x}\right)^{1 / 2} e^{(-x / 2 L)}$ with the suction parameter $s$ for different values of $M^{2}$ when $\operatorname{Pr}=1, \gamma=2$, and $\lambda=1$.

$s=0$ when $M$ was large. Meanwhile, the fourth solution profile occurred for all values of $s$ for a small value of $M^{2}$.

The velocity profile for $\lambda=1$ is depicted in Figure 8 . The magnitude of the fluid velocity at a point near the stretching sheet shows that the first solution had the lowest value whereas the third solution had the highest one. The graph of the temperature profile $\theta(\eta)$ when $\lambda=1$ is shown in Figure 9. In this set of results, the fourth solution shows the highest thermal boundary layer whereas the boundary layer thickness of the second solution is the lowest.



Figure 8. Velocity profiles when $\lambda=1$.

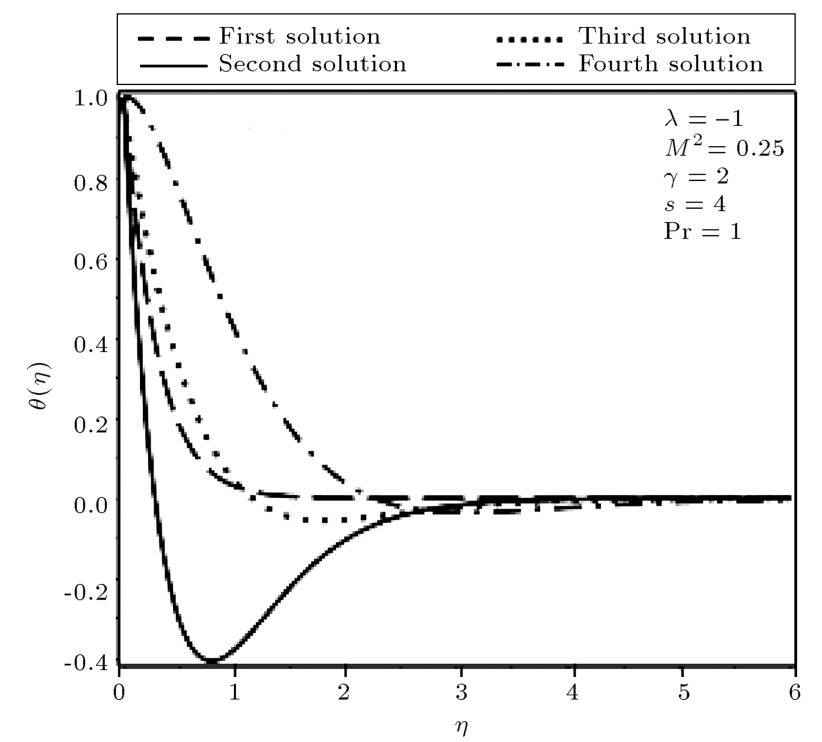

Figure 9. Temperature profiles when $\lambda=1$.

The skin friction coefficient and the local Nusselt number, with a mixed convection parameter $\lambda$ and different values of $M^{2}$, are shown in Figures 10 and 11 for $\operatorname{Pr}=1, \gamma=2$, and $s=4$. With the presence of suction, the first solution always had an increment in the skin friction coefficient and local Nusselt number caused by the effect of increasing $M^{2}$ and $\lambda$. A decrement in the value of skin friction coefficient was observed for the other profiles, due to an increment in the magnetic field, when the mixed convection parameter decreased. When examining the second and fourth solutions, the local Nusselt number became very large or very small when $\lambda$ was close to 0 . The critical value of the mixed convection parameter, $\lambda_{c}$, is the point which connects the third and fourth 


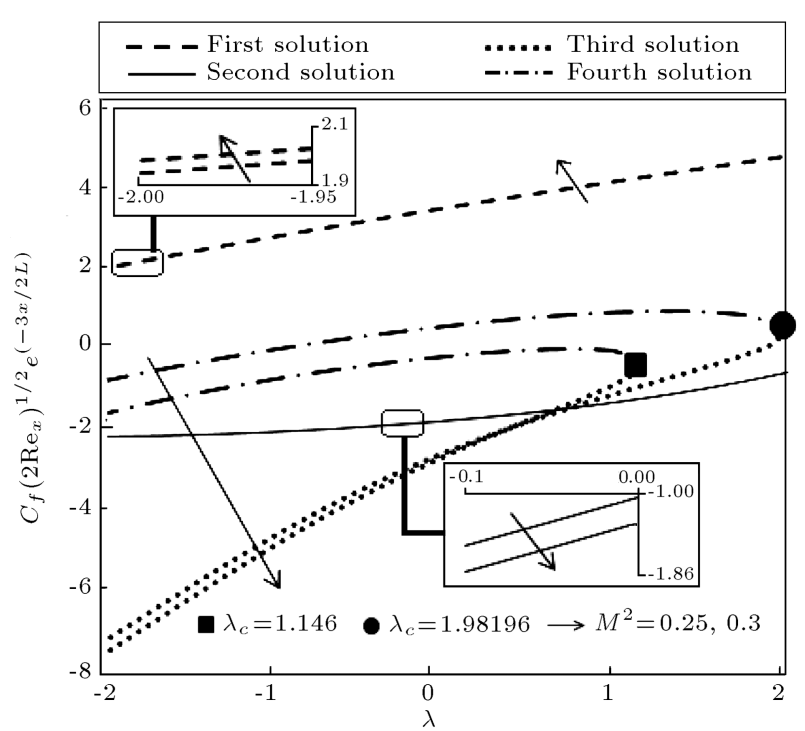

Figure 10. Variations of skin friction coefficient $C_{f}\left(2 \operatorname{Re}_{x}\right)^{1 / 2} e^{(-3 x / 2 L)}$ with mixed convection parameter $\lambda$ for different values of $M^{2}$ when $\operatorname{Pr}=1, \gamma=2$, and $s=4$.



Figure 11. Variations of local Nusselt number $\mathrm{Nu}_{x}\left(2 / \operatorname{Re}_{x}\right)^{1 / 2} e^{(-x / 2 L)}$ with mixed convection parameter $\lambda$ for different values of $M^{2}$ when $\operatorname{Pr}=1, \gamma=2$, and $s=4$.

solutions, and depends on $M^{2}$. The point $\lambda_{c}$ moves to the left for an increasing $M^{2}$. For the assisting flow situation $(\lambda>0)$, all solution profiles occurred in the range $0<\lambda<\lambda_{c}$, whereas only dual solutions (first and second solution profiles) arose for $\lambda>\lambda_{c}$. When we observed the case of opposing flow $(\lambda<0)$, all the profiles showed their existence for all values of $\lambda$. In the case of forced convection $(\lambda=0)$, only the first and third solutions appeared in the variation of heat transfer. However, the occurrence of all profiles could be seen for the variations of the skin friction coefficient when $\lambda=0$.

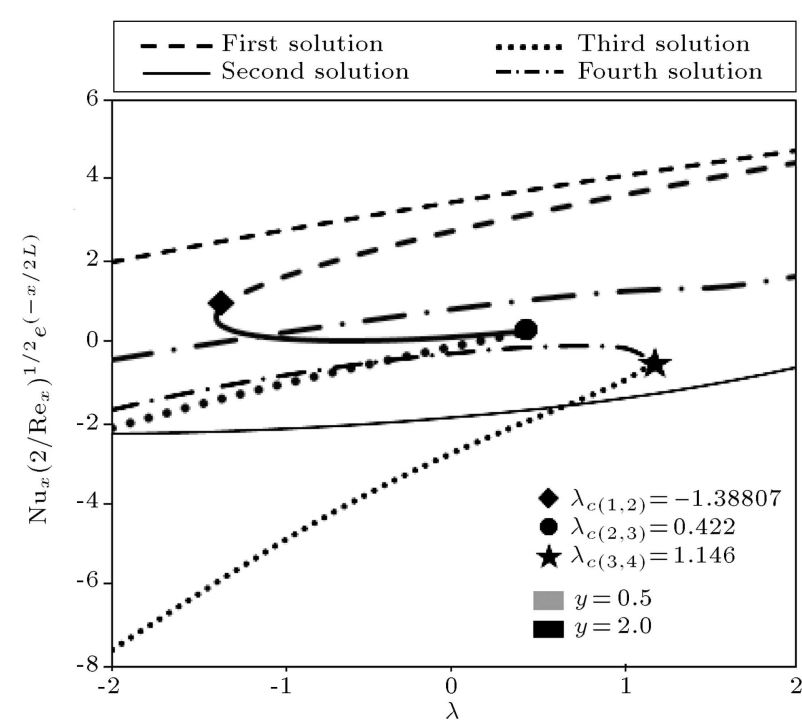

Figure 12. Variations of skin friction coefficient $C_{f}\left(2 \operatorname{Re}_{x}\right)^{1 / 2} e^{(-3 x / 2 L)}$ with mixed convection parameter $\lambda$ for different values of $\gamma$ when $\operatorname{Pr}=1, M^{2}=0.3$, and $s=4$.

Figure 12 shows illustrations of the skin friction coefficient, which depend on the change of mixed convection parameter, $\lambda$, for different rates of the Casson parameter, $\gamma(\gamma=0.5,2)$. The points of this figure were calculated numerically for $s=4$ and $M^{2}=0.3$. Foremost, all solution profiles occurred when the value of the Casson parameter was small $(\gamma=0.5)$, and their regions were in the range of $-1.38807<\lambda<0.42200$. In addition, dual solutions in $\gamma=0.5$ for opposing flow occurred when $\lambda<-1.38807$; this region involved the third and fourth solution profiles. Besides, another region of dual solutions in $\gamma=0.5$ for the assisting flow case occurred when $\lambda>0.42200$. This region of dual solutions involve first and fourth solutions. The fourth solution appeared for all values of $\lambda$ for $\gamma=0.5$. Next, when the rate of the Casson parameter increased to 2 , all solutions existed in the region of $\lambda<1.14600$. Moreover, dual solutions (first and second solutions) occurred when the assisting flow parameter was large $(\lambda>1.14600)$. In conclusion, the rate of skin friction coefficient attempted to increase for very large $\lambda$ and $\gamma$, and it was demonstrated that all the 4 solutions expanded because of increase in Casson parameter.

The local Nusselt numbers are plotted versus the mixed convection parameter as shown in Figure 13 for different values of Casson parameter, $\gamma$. When $\gamma=0.5$, all solutions came about when the values of the mixed convection parameter were $-1.38807<\lambda<0$ and $0<\lambda<0.42200$. Besides, when the values of the mixed convection parameter were $\lambda<-1.38807$, $\lambda=0$, and $\lambda>0.42200$, there existed two solution profiles at $\gamma=0.5$. These dual solutions were related to the third and fourth solutions in the opposing flow case, the first and third solutions for forced convection $\lambda=0$, and the first and fourth solutions when the 




(a)

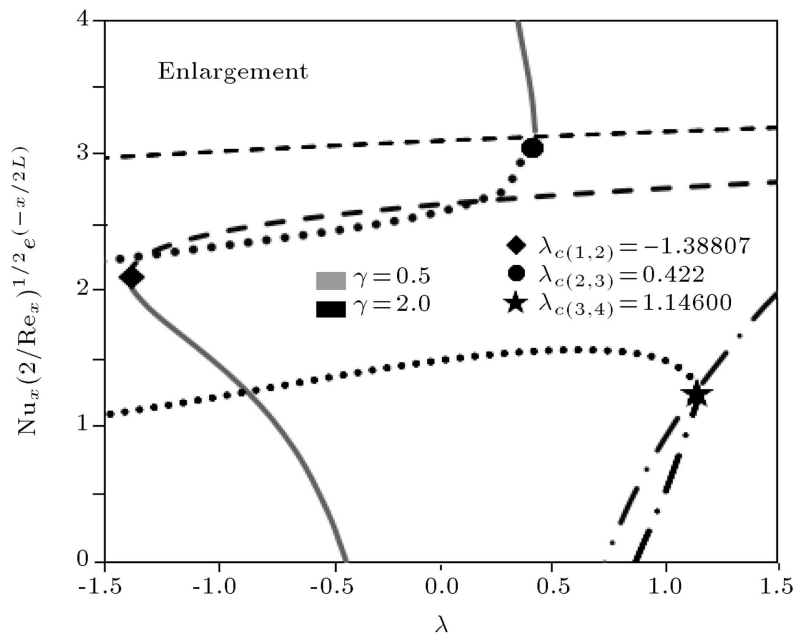

(b)

Figure 13. (a) Variations of local Nusselt number $\mathrm{Nu}_{x}\left(2 / \operatorname{Re}_{x}\right)^{1 / 2} e^{(-x / 2 L)}$ with mixed convection parameter $\lambda$ for different values of $\gamma$ when $\operatorname{Pr}=1, M^{2}=0.3$, and $s=4$. (b) Enlargement of (a).

fluid flow was assisted by a buoyancy force. When the Casson parameter $\gamma$ increased from 0.5 to 2 , all solutions became established when the values of mixed convection parameter were $\lambda<0$ and $0<\lambda<1.14600$. Furthermore, dual solutions were presented at $\gamma=2$ with the involvement of the first and second solutions when $\lambda>1.14600$. In addition, for $\gamma=2$, force convection also showed the dual solutions, which relate first and third solutions. The variation of local Nusselt number for $\gamma=2$ proved that first solution occurred for all values of $\lambda$. Conclusively, the effect of $\gamma$ for various values of $\lambda$ was to enhance the rate of the local Nusselt number for the first solution, whereas a reduction was observed with the effect of Casson rate in the third solution. However, for the assisting flow case $\lambda>0$, the second solution increased and fourth solution decreased due to the increment in the Casson parameter. In opposing flow $\lambda<0$, the two solutions (second and fourth) showed an opposite trend compared to the case when $\lambda>0$. Figure 13 also shows similar results to Figure 12 on the influence of the Casson parameter on the region of multiple solutions, which became wider when the Casson parameter became higher.

\section{Conclusions}

A detailed numerical study is performed for mixed convection MHD flow of a Casson fluid over an exponentially permeable shrinking sheet. Effects of the non-Newtonian (Casson) parameter, suction parameter, mixed convection parameter, and magnetic field parameter on the flow and heat transfer behavior are thoroughly analyzed. As already proved by Isa et al. [32], 4 solutions are obtained by containing the impact of magnetic field in mixed convection boundary layer flow of viscous Newtonian fluid over a permeable shrinking sheet. Therefore, the presence of buoyancy force, magnetic field, and suction in Casson fluid also contributes to the occurrence of multiple solutions (4 solutions). These 4 solutions exist for both the skin friction coefficient and the local Nusselt number when the flow is opposing and assisting. However, only two profiles exist for the case of forced convection, for the graph of local Nusselt number. The enlargement in the region of 4 solutions is due to the enhancement in the rate of Casson parameter. The study discloses that the quad solutions of velocity and temperature exist in certain ranges of mass suction parameter, mixed convection parameter, magnetic field parameter, and Casson parameter. Nevertheless, the effect of Casson parameter influences the rates of skin friction and local Nusselt number and changes the characteristics of Newtonian Casson fluid into those of the nonNewtonian one.

\section{Nomenclature}

$\begin{array}{ll}B(x) & \text { Magnetic field } \\ C_{f} & \text { Skin friction coefficient } \\ g & \text { Acceleration of gravity } \\ \mathrm{Gr} & \text { Grashof number } \\ L & \text { Characteristic length of the sheet } \\ M^{2} & \text { Hartman number (magnetic field } \\ & \text { parameter) } \\ \mathrm{Nu}_{x} & \text { Local Nusselt number } \\ p_{y} & \text { Yield stress of fluid } \\ \mathrm{Re} & \text { Reynolds number } \\ \mathrm{Re}_{x} & \text { Local Reynolds number } \\ T & \text { Fluid temperature } \\ T_{w} & \text { Shrinking sheet temperature }\end{array}$




$\begin{array}{ll}T_{\infty} & \text { Ambient fluid temperature } \\ U_{w} & \text { Velocity of the shrinking sheet } \\ u, v & \text { Velocity components in the } x \text { and } y \\ & \text { directions respectively } \\ v_{w}(x) & \begin{array}{l}\text { Velocity of the suction/mass transfer } \\ x, y\end{array} \quad \begin{array}{l}\text { Cartesian coordinates along the } \\ \text { surface of the sheet and normal to it }\end{array} \\ & \text { respectively }\end{array}$

\section{Greek letters}

$\begin{array}{ll}\alpha & \text { Thermal diffusivity } \\ \beta & \text { Thermal expansion coefficient } \\ \gamma & \text { Casson parameter } \\ \eta & \text { Similarity variable } \\ \theta & \text { Dimensionless temperature } \\ \lambda & \text { Mixed convection parameter } \\ \mu_{g} & \text { Plastic dynamic viscosity of the } \\ \nu & \text { non-Newtonian fluid } \\ \pi & \text { Kinematic viscosity } \\ & \text { Square of the component of the } \\ \pi_{c} & \text { deformation rate } \\ \rho & \text { Critical value of product } \\ \sigma & \text { Fluid density } \\ \tau & \text { Electrical conductivity } \\ \psi & \text { Shear rate }\end{array}$

\section{Subscripts}

c A manifestation of the critical point

$w \quad$ Shrinking sheet's state at the surface

$\infty \quad$ Free-stream condition

\section{Superscripts}

' Differentiation with respect to $\eta$

\section{References}

1. Davis, M.W. and Weber, E.J. "Liquid-liquid extraction between rotating concentric cylinders", Ind. Eng. Chem., 52(11), pp. 929-934 (1960).

2. Cohen, S. and Maron, D.M. "Experimental and theoretical study of a rotating annular flow reactor", Chem. Engng. J., 27(2), pp. 87-97 (1983).

3. Beaudoin, G. and Jaffrin, M.Y. "Plasma filtration in Couette flow membrane devices", Artificial Organs, 13(1), pp. 43-51 (1989).

4. Holeschovsky, U.B. and Cooney, C.L. "Quantitative description of ultra filtration device", AICHE J., 37(8), pp. 1219-1226 (1991).

5. Casson, N. "A flow equation for pigment oil suspension of printing ink type", In Rheology of Dispersed System, C.C. Mill, Ed., pp. 84-102, Pergamon Press, London, UK (1959).
6. Skelland, A.H.P., Non-Newtonian Flow and Heat Transfer, p. 276, John Wiley \& Sons, New York (1967).

7. Slattery, J.C., Advanced Transport Phenomena, pp. 131-142, Cambridge University Press, Cambridge (1999).

8. Rivlin, R.S. and Ericksen, J.L. "Stress deformation relations for isotropic materials", J. Rational Mech. Anal., 4(2), pp. 323-425 (1955).

9. Oldroyd, J.G. "On the formulation of rheological equations of state", Proc. R. Soc. London A, 200(1063), pp. 523-541 (1950).

10. Boyd, J., Buick, J.M. and Green, S. "Analysis of the Casson and Carreau-Yasuda non-Newtonian blood models in steady and oscillatory flow using the lattice Boltzmann method", Phys. Fluids, 19(9), pp. 93-103 (2007).

11. Goldstein, S. "On backward boundary layers and flow in converging passages", J. Fluid Mech., 21, pp. 33-45 (1965).

12. Miklavčič, M. and Wang, C.Y. "Viscous flow due to a shrinking sheet", Q. Appl Math., 64(2), pp. 283-290 (2006).

13. Mahapatra, T.R. and Nandy, S.K. "Slip effects on unsteady stagnation-point flow and heat transfer over a shrinking sheet", Meccanica, 48(7), pp. 1599-1606 (2013).

14. Wang, C.Y. "Stagnation flow towards a shrinking sheet", Int. J. Non-linear Mech., 43(5), pp. 377-382 (2008).

15. Magyari, E. and Keller, B. "Heat and mass transfer in the boundary layers on an exponentially stretching continuous surface", J. Phys. D: Appl. Phys., 32, pp. 577-585 (1999).

16. Bhattacharyya, K. "Boundary layer flow and heat transfer over an exponentially shrinking sheet", Chin Phys. Lett., 28(7), pp. 074701 (2011).

17. Sharma, R., Ishak, A., Nazar, R. and Pop, I. "Boundary layer flow and heat transfer over a permeable exponentially shrinking sheet in the presence of thermal radiation and partial slip", J. Appl. Fluid Mech., 7(1), pp. 125-134 (2014).

18. Wong, S.W., Awang, M.A.O., Ishak, A. and Pop, I. "Boundary layer flow and heat transfer over an exponentially stretching/shrinking permeable sheet with viscous dissipation", J. Aerosp. Engng., 27(1), pp. 2632 (2014).

19. Akbar, N.S., Khan, Z.H., Haq, R.U. and Nadeem, S. "Dual solutions in MHD stagnation-point flow of Prandtl fluid impinging on shrinking sheet", Appl. Math. Mech., 35, pp. 813-820 (2014).

20. Akbar, N.S., Nadeem, S., Haq, R.U. and Ye, S. "MHD stagnation point flow of Carreau fluid toward a permeable shrinking sheet: Dual solutions", Ain Shams Eng. J., 5, pp. 1233-1239 (2014). 
21. Lin, Y., Zheng, L., Zhang, X., Mad, L. and Chen, G. "MHD pseudo-plastic nanofluid unsteady flow and heat transfer in a finite thin film over stretching surface with internal heat generation", Int. J. Heat Mass Trans., 84, pp. 903-911 (2015).

22. Lin, Y., Zheng, L. and Chen, G. "Unsteady flow and heat transfer of pseudo-plastic nanoliquid in a finite thin film on a stretching surface with variable thermal conductivity and viscous dissipation", Powder Technol., 274, pp. 324-332 (2015).

23. Lin, Y., Zheng, L., Li, B. and Zhang, X. "MHD thin film and heat transfer of power law fluids over an unsteady stretching sheet with variable thermal conductivity", Therm. Sci., 20(6), pp. 1791-1800 (2014).

24. Merkin, J.H. "On dual solutions occurring in mixed convection in a porous medium", J. Eng. Math., 20, pp. 171-179 (1985).

25. Weidman, P.D., Kubitschek, D.G. and Davis, A.M.J. "The effect of transpiration on self-similar boundary layer flow over moving surfaces", Int. J. Eng. Sci., 44, pp. $730-737$ (2006).

26. Paullet, J. and Weidman, P. "Analysis of stagnation point flow toward a stretching sheet", Int. J. Nonlinear Mech., 42, pp. 1084-1091 (2007).

27. Harris, S.D., Ingham, D.B. and Pop, I. "Mixed convection boundary-layer flow near the stagnation point on a vertical surface in a porous medium: Brinkman model with slip", Transport Porous Med., 77, pp. 267285 (2009).

28. Postelnicu A. and Pop, I. "Falkner-Skan boundary layer flow of a power-law fluid past a stretching wedge", Appl. Math. Comput., 217, pp. 4359-4368 (2011).

29. Rosca, A.V. and Pop, I. "Flow and heat transfer over a vertical permeable stretching/shrinking sheet with a second order slip", Int. J. Heat Mass Tran., 60, pp. 355-364 (2013).

30. Rohni, A.M., Ahmad, S., Ismail A.I.M. and Pop, I. "Boundary layer flow and heat transfer over an exponentially shrinking vertical sheet with suction", Int. J. Therm. Sci., 64, pp. 264-272 (2013).

31. Rohni, A.M., Ahmad, S. and Pop, I. "Flow and heat transfer at a stagnation-point over an exponentially shrinking vertical sheet with suction", Int. J. Therm. Sci., 75, pp. 164-170 (2014).

32. Isa, S.S.P.M., Arifin, N.M., Nazar, R., Bachok, N., Ali, F.M. and Pop, I. "Effect of magnetic field on mixed convection boundary layer flow over an exponentially shrinking vertical sheet with suction", Int. J. Mech., Aerosp., Ind. Mechatron. Eng., 8(9), pp. 1509-1514 (2014).

33. Bhattacharyya, K., Vajravelu, K. and Hayat, T. "Slip effects on the parametric space and the solution for boundary layer flow of Casson fluid over a porous stretching/shrinking sheet", Int. J. Fluid Mech. Res., 40(6), pp. 482-493 (2013).
34. Bhattacharyya, K., Hayat, T. and Alsaedi, A. "Analytic solution for magnetohydrodynamic boundary layer flow of Casson fluid over a stretching/shrinking sheet with wall mass transfer", Chin. Phys. B, 22, Article ID 024702 (2013).

35. Bhattacharyya, K., Hayat, T. and Alsaedi, A. "Exact solution for boundary layer flow of Casson fluid over a permeable stretching/shrinking sheet", ZAMM, 94(6), pp. 522-526 (2014).

36. Wahiduzzaman, M., Miah, M.M., Hossain, M.B., Johora, F. and Mistri, S. "MHD Casson fluid flow past a non-isothermal porous linearly stretching sheet", Prog. Nonlinear Dynam. Chaos, 2, pp. 61-69 (2014).

37. Nadeem, S., Haq, R.U., Akbar, N.S. and Khan, Z.H. "MHD three-dimensional Casson fluid flow past a porous linearly stretching sheet", Alexandria Eng. J., 52, pp. 577-582 (2013).

38. Nadeem, S., Haq, R.U. and Akbar, N.S. "MHD threedimensional boundary layer flow of Casson nanofluid past a linearly stretching sheet with convective boundary condition", IEEE Trans. Nanotechnol., 13, pp. 109-115 (2014).

39. Ramachandra Prasad, V., Subba Rao, A. and Anwar Bég, O. "Flow and heat transfer of Casson fluid from a horizontal circular cylinder with partial slip in nonDarcy porous medium", J. Appl. Computat. Math., 2, Article ID 1000127 (2013).

40. Makanda, G., Shaw, S. and Sibanda, P. "Effects of radiation on MHD free convection of a Casson fluid from a horizontal circular cylinder with partial slip in non-Darcy porous medium with viscous dissipation", Boundary Value Problems, 2015, Article ID 75 (2015).

41. Pramanik, S. "Casson fluid flow and heat transfer past an exponentially porous stretching sheet in presence of thermal radiation", Ain Shams Eng. J., 5, pp. 205-212 (2014).

42. Raju, C.S.K., Sandeep, N., Sugunamma, V., Jayachandra Babu, M. and Ramana Reddy, J.V. "Heat and mass transfer in magnetohydrodynamic Casson fluid over an exponentially permeable stretching surface", Eng. Sci. Technol., Int. J., 19(1), pp. 45-52 (2016).

43. Animasaun, I.L., Adebile, E.A. and Fagbade, A.I. "Casson fluid flow with variable thermo-physical property along exponentially stretching sheet with suction and exponentially decaying internal heat generation using the homotopy analysis method", J. Nigerian Math. Soc., 35(1), pp. 1-17 (2016).

44. Nadeem, S., Haq, R.U. and Lee, C. "MHD flow of a Casson fluid over an exponentially shrinking sheet", Scientia Iranica B, 19(6), pp. 1550-1553 (2012).

45. Haq, R.U., Nadeem, S., Khan, Z.H. and Okedayo, T.G. "Convective heat transfer and MHD effects on Casson nanofluid flow over a shrinking sheet", CEJP, 12, pp. 862-871 (2014).

46. Nakamura, M. and Sawada, T. "Numerical study on the flow of non-Newtonian fluid through an axisymmetric", J. Biomec. Engng., 110(2), pp. 137-143 (1988). 


\section{Biographies}

Siti Suzilliana Putri Mohamed Isa has been a tutor of Mathematics since July 2012 at the Centre of Foundation Studies for Agricultural Science, UPM, Malaysia. She received her BSc in Physics in 2007 and her MSc degree in 2010 in the field of Mathematical Sciences and Applications (Fluid Dynamics). Currently, she is a PhD Candidate in the field of Fluid Dynamics at the Institute for Mathematical Research in UPM, Malaysia. During her PhD, she is working on steady- and unsteady-convection boundary layer flow on a stretching/shrinking sheet in viscous and Casson fluids.

Norihan Md. Arifin is Associate Professor of Mathematics in the Department of Mathematics, UPM, Malaysia. She received her MSc in Industrial Mathematics from University of Strathclyde-Glasgow, UK, in 1996 and her PhD in Mathematics from UKM, Malaysia. Her research interests include fluid mechanics and heat transfer with application to the thermal convection and boundary-layer theory, and heat transfer in Newtonian and non-Newtonian as well as in fluid saturated porous media and nanofluids.

Roslinda Nazar has been a Professor of Mathematics, since January 2012, in the School of Mathematical Sciences, Faculty of Science and Technology, UKM, Malaysia. She obtained the BSc in Mathematics from the University of Illinois at Urbana-Champaign, Illinois, USA, in January 1995. Later, in October 1996, she earned the MSc in Mathematics of Nonlinear Models at the Heriot-Watt University \& University of Edinburgh, Scotland, UK. She received the $\mathrm{PhD}$ in Applied Mathematics in January 2004 in the field of Fluid Dynamics and Heat Transfer from the Universiti Teknologi Malaysia, Skudai, Johor, Malaysia. Her area of research covers fluid dynamics, heat transfer, and mathematical modelling.

Norfifah Bachok is currently Associate Professor in the Department of Mathematics, UPM, Malaysia. She is the head of program: Mathematical Physics and Engineering at the Institute for Mathematical Research, UPM. Her expertise covers fluid flow, heat transfer, and boundary layer theory.

Fadzilah Md Ali is a Senior Lecturer in the Department of Mathematics, UPM, Malaysia. Her major research areas include fluid mechanics and heat transfer in boundary layer.

Ioan Pop is a Professor of Applied Mathematics in the Faculty of Mathematics and Computer Science, Babeş-Bolyai University, Romania. His fields of interest include fluid mechanics and heat transfer with application to boundary-layer theory, heat transfer in Newtonian and non-Newtonian fluids, convective flow in fluid-saturated porous media, and magnetohydrodynamics. 